\title{
ENHANCED MICROSTRUCTURE AND MECHANICAL PROPERTIES OF SiC PARTICLE REINFORCED ALUMINIUM ALLOY COMPOSITE MATERIALS
}

\author{
Manoj Kumar Pal ${ }^{1 *}$, Arnav Vikram ${ }^{2)}$, Vineet Bajaj ${ }^{3)}$ \\ 1) Institute of Physical Metallurgy, Metal Forming, and Nanotechnology, University of Miskolc, \\ Hungary-3515 \\ 2) Department of Mechanical Engineering, IST, Delhi-NCR Campus, Modinagar, India-201204 \\ 3) Department of Civil Engineering, SRM University, Delhi-NCR, Sonipat, Haryana, India- \\ 131029
}

Received: 21.06 .2019

Accepted: 24.09.2019

'Corresponding author: e-mail: manojsantosh2002@gmail.com Tel.: +36204467103, Institute of Physical Metallurgy, Metal Forming, and Nanotechnology, University of Miskolc, Hungary

\begin{abstract}
Aluminium6061 alloy composites containing various volume fractions of Silicon Carbide ( $\mathrm{SiC}$ ) particles $(0,5 \%, 10 \%, 15 \%, 20 \%$ and $25 \%)$ were prepared by stir casting method. In the current study, microstructures and mechanical properties of cast silicon carbide (SiC) reinforced aluminum matrix composites (AMCs) were investigated. Optical microscopic examination, SEM, tensile strength test, hardness test and elongation test were carried out. The results showed that with the addition of $\mathrm{SiC}$ reinforcements in Aluminum6061 matrix increased hardness and tensile strength however, decreased elongation at $25 \% \mathrm{SiC}$ reinforced AMC. Hardness and tensile strength were observed to be are maximum at $25 \% \mathrm{SiC}$ and elongation is minimum at $25 \% \mathrm{SiC}$. Microstructural observation confirmed clustering and homogeneous distribution of SiC particles in the Al6061 matrix.
\end{abstract}

Keywords: Metal Matrix Composites, Aluminium Alloy 6061, Silicon Carbide, SEM

\section{Introduction}

High strength, low specific density and good wear resistance are the main keys of the Aluminium matrix composites with incorporation of fourth alloying materials fibers or particles and whiskers. The metal reinforced aluminium matrix composites are economical but also have good mechanical properties [1]. Many methods are available for fabrication of particulate reinforced metal matrix composites (MMCs) such as powder metallurgy [1, 2], casting method [3], squeeze casting [4] and compo-casting.

Several researchers have reported that the use of hard particles $\left(\mathrm{Al}_{2} \mathrm{O}_{3}\right.$ or $\left.\mathrm{SiC}\right)$ in aluminum alloys improve the mechanical properties of these alloys [5]. Tensile strength, hardness and elongation are main big problem for aluminium alloy matrix. There is no general relationship established between mechanical properties (Tensile strength, hardness and elongation) microstructural factors and processing parameters. Recent studies revealed that the strength of matrix interface and mechanical properties of matrix materials affected by the addition in the volume fraction, and spatial distribution of second phase particles [6].

A non-metallic ceramic such as $\mathrm{SiC}, \mathrm{Al}_{2} \mathrm{O}_{3}, \mathrm{SiO}_{2}, \mathrm{~B}_{4} \mathrm{C}, \mathrm{Al}-\mathrm{N}$ are used in an AMC matrix because of pure aluminium or an alloy of it. Aluminium alloys are used in AMC due to high corrosion resistivity, high damping capacity, low density and good electrical and thermal conductivities [7]. $\mathrm{SiC}$, Boron and $\mathrm{Al}_{2} \mathrm{O}_{3}$ particles are affected in tensile strength, hardness and elongation behavior 
of MMCs. However, a limited number of researches have been reported on the abrasion behavior of ceramic particle reinforced aluminium alloy composites [1].

The purpose of the current study is to: (a) produce particle-reinforced metal matrix composites by stir casting method and; (b) measure the density, hardness and ultimate tensile strength, elongation and microstructure of the composites.

\section{Experimental Procedures}

Aluminum metal matrix composites were prepared at various volume fractions of SiC particles fabricated by stir casting method. Aluminium6061 alloy was used as the matrix material and $\mathrm{SiC}$ particles were used as the reinforcement material. The $\mathrm{SiC}$ in the experiments was supplied by Norton Co. The composite was prepared by stir casting method for fabrication which gives the uniform distribution of reinforcement particle. Silicon carbide, which is used as a mesh with the particle size of $200 /+300$ (particle size is below $74 \mu \mathrm{m}$ and above $53 \mu \mathrm{m}$ ). It is a very fine powder in appearance and has high hardness value therefore; silicon carbide is used for reinforcement purpose in powdered form. In the present study, the chemical composition of Aluminium 6061 alloy used is presented in Table 1.

Table 1 Nominal Chemical Composition of Aluminium Alloy (Al6061)

\begin{tabular}{|c|c|c|c|c|c|c|c|c|c|c|c|c|}
\hline Elements & $\mathbf{S i}$ & $\mathbf{C u}$ & $\mathbf{N i}$ & $\mathbf{Z n}$ & $\mathbf{S n}$ & $\mathbf{M n}$ & $\mathbf{M g}$ & $\mathbf{P b}$ & $\mathbf{F e}$ & $\mathbf{C r}$ & $\mathbf{T i}$ & $\mathbf{A l}$ \\
\hline $\boldsymbol{\%}$ & 0.43 & 0.24 & 0.05 & 0.25 & 0.001 & 0.139 & 0.802 & 0.24 & 0.7 & 0.25 & 0.15 & Balance \\
\hline
\end{tabular}

In this process of fabrication, a dispersed phase and a molten metal matrix are mixed together with the help of mechanical stirring. Firstly, Aluminium6061 is taken in the form of plates. These plates were then placed in a graphite crucible chamber and heated using the electrical resistance furnace. The temperature of the furnace is set at $750^{\circ} \mathrm{C}$. Once Aluminium 6061 is completely melted, silicon carbide which is in form of powder was poured into the same crucible chamber with different percentages and temperature was gradually increased to $1000^{\circ} \mathrm{C}$. While pouring the reinforcing material into the matrix material; a constant speed of $300 \mathrm{rpm}$ with the help of mechanical stirrer was maintained to create a vortex. The stir was carried out for approximately 30 minutes to obtain a uniform mixture. Afterwards, molten MMC is poured into a sand cast to obtain MMC pipe. The cast was quenched with water in order to cool down MMC quickly. This procedure was repeated for silicon carbide at various volume fractions of 5\%, 10\%, 15\%, 20\% and 25\% [6, 8, 9, 10].

To investigate the microstructure of the specimens, 1000 and 1200 grit papers were used in grinding for preparing the specimen surfaces. Then $3 \mu \mathrm{m}$ diamond paste was used for the polishing [10]. Etching was done with the help of Keller's reagent in order to conduct micro structural testing and the mechanical characterization. The etched specimen was observed under both, optical microscope as well as scanning electron microscope.

\section{Results and Discussion}

\subsection{Microstructure}

The grain size of the aluminium composites was smaller than that of the aluminum alloy and the grain size of composites decreases with the increase of volume fraction of SiC. It was observed that major portion of $\mathrm{SiC}$ particles segregated along the grain boundaries. At the melting stage and the solidification stage, the microstructures of the composites were investigated. The ultrasonic treatment dispersed $\mathrm{SiC}$ particles at the melting stage [9]. 


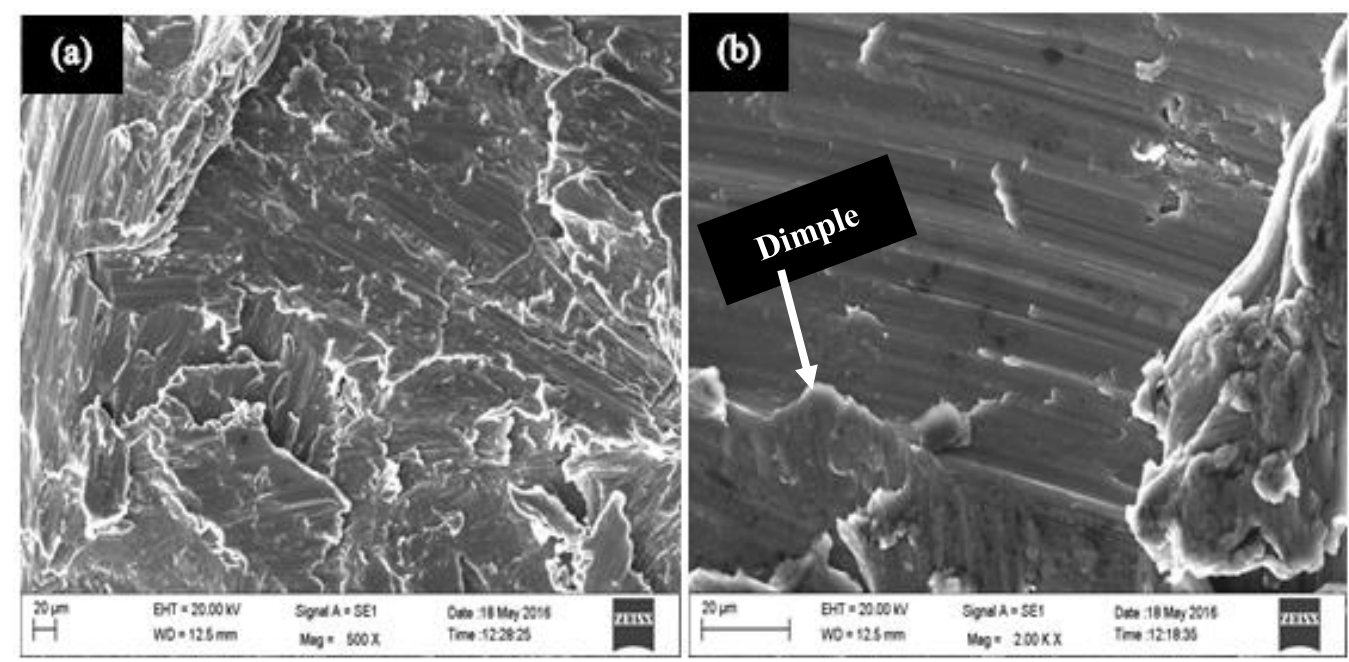

Fig. 1 SEM micrograph (a) Distribution of A16061/5\%SiC composite (b) Fractured surface of Al6061/10\% SiC composite
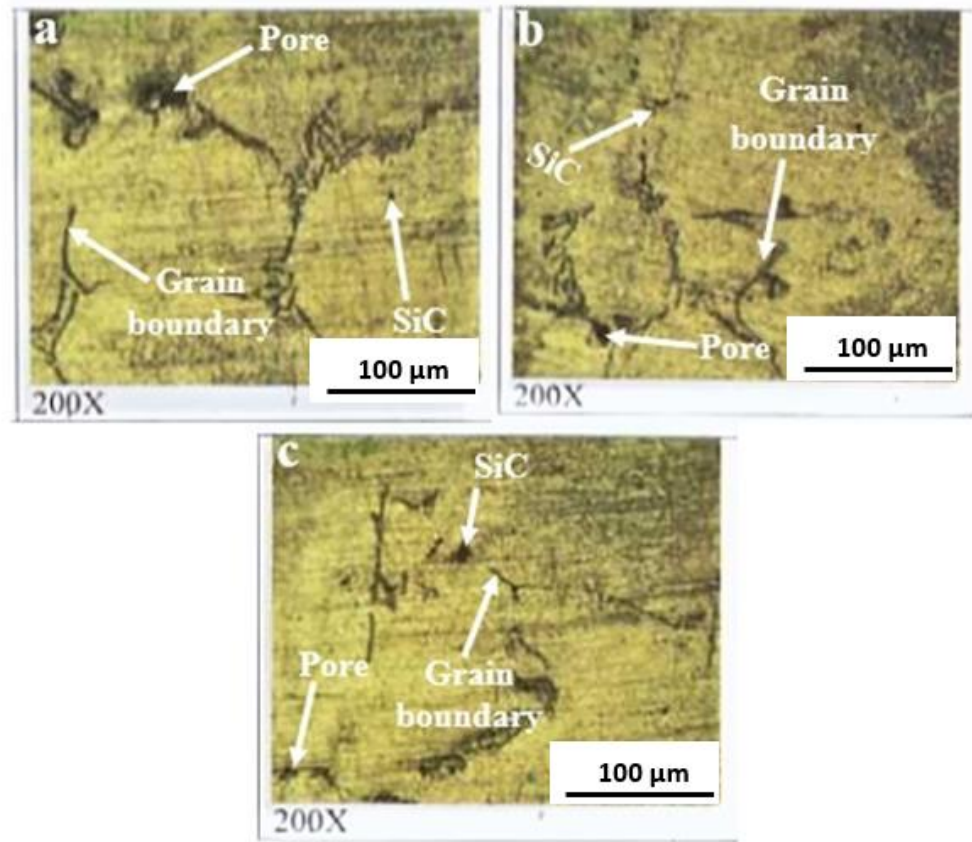

Fig. 2 Optical images (a) Al6061/5\% SiC composite (b) Al6061/10\% SiC composite (c) Al6061/15\% SiC

It is clearly observed that most of $\mathrm{SiC}$ particles are dispersed in the aluminium i.e. uniform distribution of the $\mathrm{SiC}$ on each composite particle surface. When composite powders are used for the melting, Al6061 start to melt, firstly on the outer surface and then on from the inside while gradually releasing the $\mathrm{SiC}$ particles into the molten mix which is evident from a good distribution in the melt [11]. 
Fig. 1 depicts SEM fracture surfaces of the composite with the volume fraction of the SiC particles of 5\% and 10\%, respectively. A typical ductile fracture can be observed in the Fig. 1 with numerous dimples over the entire surface are visible and it shows both the ductile and brittle fracture. All these fracture surfaces have dimples in the matrix and de-cohesion of SiC particles in the matrix. The reason of dimples is the void nucleation and subsequent coalescence because of a strong shear deformation and fracture on the shear plane are the primary reasons for cause of such dimples. It can also be seen that increasing the volume fraction of the $\mathrm{SiC}$ particles decreases the ductility of the material while it increases the brittle behavior of material. This is the major difference between the fracture surfaces $[12,13]$.

Fig. 2 shows the optical microstructures of the composites with 5\%,10\% and $15 \%$ volume fractions of the $\mathrm{SiC}$ particles. It is evident from the Fig. 2 shows that 5\%, 10\% and 15\% volume fractions of the $\mathrm{SiC}$ particles are uniformly distributed in the $\mathrm{Al}$ matrix. Also, $\mathrm{SiC}$ pores and grain boundaries can be observed in the aluminum composites as evident in Fig. 2, the pores are evident and there is an increase in the pore percentage with an increase in the $\mathrm{SiC}$ particles which indicates that the interfacial cohesion is weak between the $\mathrm{SiC}$ particles and matrix [9].

\subsection{Mechanical properties}

Table 2 shows the mechanical properties of the composite as a function of the volume fraction of the $\mathrm{SiC}$ particles. It also illustrates that with an increase in the volume fraction of the $\mathrm{SiC}$ particles, there is an increase in both tensile strength as well as hardness. While the elongation decreases with an increase in the volume fraction of the $\mathrm{SiC}$ particles. Increase in the strength of the composites but degradation the plasticity of the composites with increase in $\mathrm{SiC}$ particles has been reported earlier $[14,15,16]$.

Table 2 Mechanical properties of composites (Al6061/vol\% SiC)

\begin{tabular}{|c|c|c|c|}
\hline $\begin{array}{c}\text { Volume fraction of } \\
\mathrm{SiC} \text { particles }(\%)\end{array}$ & $\begin{array}{c}\text { Tensile } \\
\text { Strength }(\mathrm{MPa})\end{array}$ & $\begin{array}{c}\text { Vickers Hardness } \\
(\mathrm{VH})\end{array}$ & $\begin{array}{c}\text { Elongation } \\
(\%)\end{array}$ \\
\hline 0 & 82 & 23.50 & 24.3 \\
\hline 5 & 93 & 37.25 & 15.2 \\
\hline 10 & 99.5 & 42.3 & 11.4 \\
\hline 15 & 113 & 44.10 & 9.1 \\
\hline 20 & 128 & 46.23 & 6.9 \\
\hline 25 & 145 & 47.3 & 5.7 \\
\hline
\end{tabular}

In general, a decrease in the plasticity but an increase in the strength of composites with addition of $\mathrm{SiC}$ particles can be related to formation of micro crack formed during deformation. Also SiC has high strength but is brittle in nature. It was observed that two types of the micro cracks were initiated by the addition of $\mathrm{SiC}$ particles. First interfacial cohesion is strong while the second interfacial cohesion is weak between $\mathrm{SiC}$ and matrix. In first condition, at high stress concentration, the local strain and dislocation density reached the critical values and the $\mathrm{SiC}$ particles fractured to nucleate micro-cracks while in second condition, before the SiC particles could fracture, the $\mathrm{SiC}$ particles and matrix happened to nucleate micro-cracks. SONG et al [9] reported similar kind of results from 2008 to 2015.

Table 2 shows the experimental data and it can be seen from the data that elongation of the composites decreases with the volume fraction of the SiC particles. Decrease in the elongation of the composites can be achieved by the decrease in the dislocation slip distance with the increase in the volume fraction of $\mathrm{SiC}$ particles [10]. 
Fig. 1 shows a typical SEM image of the fabricated composite with 5\% and 10\% SiC volume fraction. In this composite, pores are uniformly distributed in the matrix. Appearance of pores in the matrix (due to the low die-pressing pressure) reduced the plasticity of the composites and is predominant in initiation of the micro crack formed during deformation [17, 18].

There is an increase in the average hardness of composite with an increase the amount of $\mathrm{SiC}$ particulate. But this $\mathrm{SiC}$ can be allocated to (a) the presence of harder $\mathrm{SiC}$ particles and (b) during indentation, higher restrictions to the localized matrix deformation. So the presence of stronger and stiffer particles accommodate in preventing localized plastic deformation of the matrix during micro-hardness indentation [19].

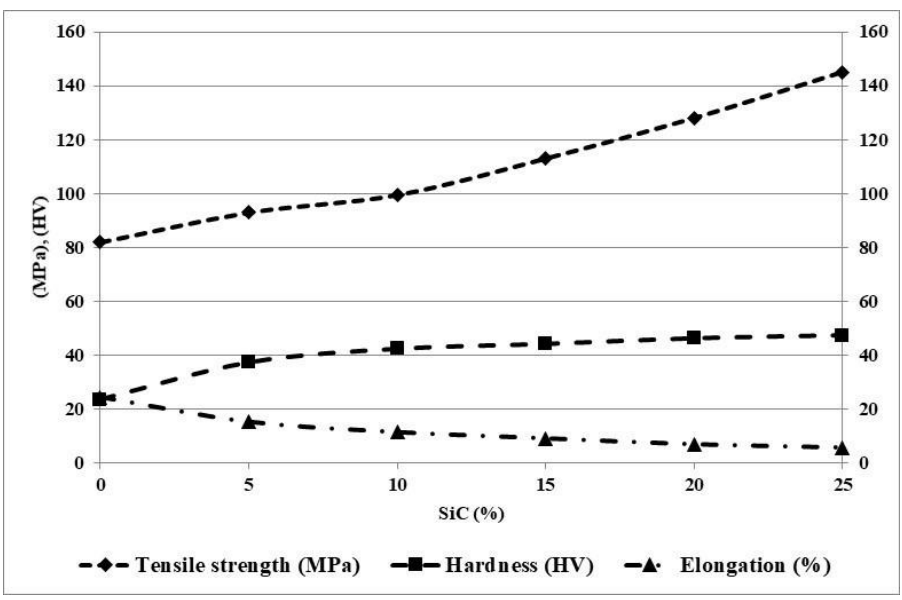

Fig. 3 Mechanical properties of composites (A16061/vol\% SiC)

The micro hardness of composite means the interface bonding between reinforcing particles and matrix. Table.1 shows the Vickers hardness values of composite for varying volume fraction of $\mathrm{SiC}$ reinforcement. Table.1 also shows the addition of $\mathrm{SiC}$ particles in Al6061 matrix composites and Fig. 3 shows that the hardness is increased with an increase in volume fraction of SiC. The presence of harder and well bonded $\mathrm{SiC}$ particles in $\mathrm{Al}$ matrix that impede the movement of dislocations increases the hardness of AMCs [20, 21].

\section{Conclusions}

Al6061 with varying $\mathrm{SiC}$ content $(0 \%, 5 \%, 10 \%, 15 \%, 20 \%$ and $25 \%$ volume fraction) were prepared by stir casting method. Microstructure and mechanical properties (hardness, tensile strength and elongation) characteristics of the prepared composites were investigated. Based on experimental evaluation, following conclusions are evaluated:

1. A stir casting route was developed for the metal matrix composites by adding SiC particles. Various sizes of MMCs could be produced successfully by stir casting method consisting of $10 \%$ and $25 \%$ volume fraction of $\mathrm{SiC}$ particles.

2. Clustering and homogenous distribution of SiC particles in Al6061 matrix were observed in the microstructures.

3. The increase in compressive strength hardness of composite due to the addition of SiC particles because $\mathrm{SiC}$ is harder than Al6061 matrix.

4. Mechanical properties (tensile strength and Vickers hardness) of composites increased and elongation is decreased as increased of volume fraction of SiC. Fig. 3 confirmed that 
tensile strength and Vickers hardness are maximum 25\% SiC content and elongation is lower at $25 \% \mathrm{SiC}$ content AMC.

\section{References}

[1] M. O. Bodunrin, K. K. Alaneme, L. H. Chown: J. Mater. Res. Technol., Vol. 4, 2015, p. 434445, https://doi.org/10.1016/j.jmrt.2015.05.003

[2] M. K. Pal, G. Gergely, D. K. Horvath, Z. Gacsi: Arch. Metall. Mater., Vol. 64, No. 2, 2019, p. 603-606, http://dx.doi.org/10.24425/amm.2019.127585

[3] M. K. Pal, G. Gergely, D. K. Horvath, Z. Gacsi: Powder Metallurgy Progress, Vol. 18, No. 1, 2018, p. 049-057, http://dx.doi.org/10.1515/pmp-2018-0006

[4] M. T. Sijo, K. R. Jayadevan: Procedia Technol., Vol. 24, 2016, p. 379-385, https://doi.org/10.1016/j.protcy.2016.05.052

[5] S. Gopalakrishnan, N. Murugan: Compos. Part B Eng., Vol. 43, No. 2, 2012, p. 302-308, https://doi.org/10.1016/j.compositesb.2011.08.049

[6] Y. Sahin: Materials \& Design, Vol. 24, 2003, p. 671-679, https://doi.org/10.1016/S02613069(03)00156-0

[7] H. Kala, K. K. S. Mer, S. Kumar: Procedia Mater. Sci., Vol. 6, 2014, p. 1951-1960, https://doi.org/10.1016/j.mspro.2014.07.229

[8] H. Uzun: Mater. Des., Vol. 28, 2007, p. 1440-1446, https://doi.org/10.1016/j.matdes.2006.03.023

[9] M. Song and B. Huang: Mater. Sci. Eng. A, Vol. 488, 2008, p. 601-607, https://doi.org/10.1016/j.msea.2008.03.022

[10]H. R. Ezatpour, S. A. Sajjadi, M. H. Sabzevar, Y. Huang: Mater. Des., Vol. 55, 2014, p. $921-$ 928, https://doi.org/10.1016/j.matdes.2013.10.060

[11]L. J. Zhang, F. Qiu, J. G. Wang, Q. C. Jiang: Mater. Sci. Eng. A, Vol. 626, 2015, p. 338-341, https://doi.org/10.1016/j.msea.2014.12.089

[12] M. J. Shen, X. J. Wang, T. Ying, K. Wu, W. J. Song: J. Alloys Compd., Vol. 686, 2016, p. 831-840, https://doi.org/10.1016/j.jallcom.2016.06.232

[13]M. Kok: J. Mater. Process. Technol., Vol. 161, 2005, p. 381-387, https://doi.org/10.1016/j.jmatprotec.2004.07.068

[14] M. H. Rahman, H. M. M. Al Rashed: Procedia Eng., Vol. 90, 2014, p. 103-109, https://doi.org/10.1016/j.proeng.2014.11.821

[15] N. Parvin, R. Assadifard, P. Safarzadeh, S. Sheibani, P. Marashi: Mater. Sci. Eng. A, Vol. 492, 2008, p. 134-140, https://doi.org/10.1016/j.msea.2008.05.004

[16] H. Abdizadeh, R. Ebrahimifard, M. A. Baghchesara: Compos. Part B Eng., Vol. 56, 2014, p. 217-221, https://doi.org/10.1016/j.compositesb.2013.08.023

[17]R. Taherzadeh Mousavian, R. Azari Khosroshahi, S. Yazdani, D. Brabazon, A. F. Boostani: Mater. Des., Vol. 89, 2016, p. 58-70, https://doi.org/10.1016/j.matdes.2015.09.130

[18] G. B. Veeresh Kumar, C. S. P. Rao, N. Selvaraj: Compos. Part B Eng., Vol. 43, 2012, p. 1185-1191, https://doi.org/10.1016/j.compositesb.2011.08.046

[19]X. L. Zhong, M. Gupta: Journal of Physics D, Vol. 41, 2008, p. 1-7, http://iopscience.iop.org/0022-3727/41/9/095403

[20] K. C. Mohanakumara, H. Rajashekar, S. Ghanaraja, S. L. Ajitprasad: Procedia Mater. Sci., Vol. 5, 2014, p. 934-943, https://doi.org/10.1016/j.mspro.2014.07.381

[21]X. J. Wang et al.: Mater. Des., Vol. 57, 2014, p. 638-645, https://doi.org/10.1016/j.matdes.2014.01.022 